\title{
Brain Decoding Based on Functional Magnetic Resonance Imaging Using Machine Learning: A Comparative Study
}

\author{
Jeiran Choupan, Julia Hocking, Kori Johnson, David Reutens, and Zhengyi Yang
}

\begin{abstract}
Brain decoding of functional Magnetic Resonance Imaging data is a pattern analysis task that links brain activity patterns to the experimental conditions. Classifiers predict the neural states from the spatial and temporal pattern of brain activity extracted from multiple voxels in the functional images in a certain period of time. The prediction results offer insight into the nature of neural representations and cognitive mechanisms and the classification accuracy determines our confidence in understanding the relationship between brain activity and stimuli. In this paper, we compared the efficacy of three machine learning algorithms: neural network, support vector machines, and conditional random field to decode the visual stimuli or neural cognitive states from functional Magnetic Resonance data. Leave-one-out cross validation was performed to quantify the generalization accuracy of each algorithm on unseen data. The results indicated support vector machine and conditional random field have comparable performance and the potential of the latter is worthy of further investigation.
\end{abstract}

Index Terms-Brain decoding, functional magnetic resonance imaging, neural network, support vector machine, conditional random field.

\section{INTRODUCTION}

Functional Magnetic Resonance Imaging (Functional MRI, or fMRI) measures Blood Oxygen Level Dependent (BOLD) signal, which is the result of neural activity in the corresponding part of the human brain. Each fMRI scan produces a three dimensional image of the whole brain activities every few seconds, with a spatial resolution of a few millimeters with respect to various stimulus received by the subjects [1]. Functional MRI provides a non-invasive and large-scale view of human cortical activity that can contribute for specifying patterns of neural activity. Establishing a corresponding map between cortical activation patterns and different human brain cognition states is a challenging task [2] since 1) fMRI signals are noisy and non-stationary in time, 2) Each voxel represents responses influenced by hundreds of thousands of neurons [3].

Many studies report applications of brain decoding from task fMRI (TfMRI). In the area of vision science are on neural processes underlying low level visual aftereffect [4], predicting conscious visual perceptions [5], dissociating brain areas responsive to biological motion [6], enabling communication with patients in minimally conscious states [7], and distinguishing brain states underlying face matching

Manuscript received November 25, 2012; revised January 14, 2013.

The authors are with the University of Queensland, Brisbane, Australia (e-mail: j.choupan@uq.edu.au;

kori.johnson@cai.uq.edu.au; j.hocking@uq.edu.au;

steven.yang@itee.uq.edu.au ). and location matching tasks [8].

Classification of brain activity patterns specifies a causal relationship between experimental stimuli and pattern of neural activity in different brain regions. Classification methods use a subset of brain voxel responses as training data in order to define a decision boundary in the space of brain voxels response patterns. They are different in 1) the shape of the decision boundary and 2) the way the boundary is placed on the basis of the training data. Decision boundaries can be hyperplanes in linear classifiers, or more complex nonplanar boundaries in nonlinear classifiers. For example, the decision boundary in linear Support Vector Machine (SVM) is a hyperplane, which has the maximum margin to the voxel patterns on either side. In practice, the classification performance in different methods depends on how well the classifier's implicit assumptions hold in the domain in which it is applied [9].

In previous studies the performance of different classification methods were evaluated for fMRI data. Cox and Savoy [10] compared the classification performance of linear and polynomial SVM to classify fMRI response patterns into different categories of visual stimulus. They found that linear SVM performs superior to polynomial SVM and the latter has overfitting problem. In another study, results of decoding block-design fMRI data using linear SVM were better than nonlinear SVM [11]. A comparison study on Gaussian naïve Bayes (GNB), SVM, and k-nearest-neighbors (KNN) classifiers applied to three different data sets suggested that GNB and SVM performed almost equally [12] and KNN was always inferior to GNB and SVM [9].

It is not clear that which machine learning method performs is better than others in case of different types of tasks. However, SVM appeared to have an edge over the other classifiers across different tasks [13].

Recently, Conditional Random Field (CRF) based classification techniques attracted research interests in signal and image processing and fMRI study, which offers several advantages over other probabilistic methods such as hidden Markov and maximum entropy Markov models [14, 15]. CRF has been utilized to constrain the search space of brain regions with significant activities corresponding to experimental stimuli or cognitive states [16]. A CRF model considering the time delay of BOLD signal was proposed in [17]. They compared CRF with several other classifiers on predicting mental states from functional MRI images and found that CRF performed best in 5 out of 6 subjects. The whole brain was segmented into anatomical regions and the signs of average voxel BOLD signals in each region were used as the input variables. Voxel-level pattern analysis, however, was not attempted. 
In this study, we applied three machine learning techniques on two different fMRI datasets to compare their decoding performance. NN, SVM, and CRF were chosen as the machine learning methods and integrated into a multiple voxel pattern analysis framework to decode the brain tasks/states related fMRI data. We picked one publicly available dataset and our own dataset for performance comparison using Leave-one-out cross-validation (LOOCV). The results show that while SVM has the best overall performance, CRF is superior in some cases implying its potential application.

\section{MATERIALS AND METHODS}

Two datasets used in this study are: the canonical dataset shipped with the Multiple Voxel Pattern Analysis (MVPA) Toolbox developed in the Princeton Neuroscience Institute [18], and a task-related fMRI dataset using visual semantic stimuli acquired as part of an epilepsy project. The two datasets are called as the Haxby dataset and VisSemantic dataset for short in this paper, respectively.

In the Haxby dataset, the fMRI images were acquired when eight categories of object (faces, houses, cats, bottles, scissors, shoes, chairs, and scrambled control stimuli) were displayed to ten participants and more information on the dataset can be found on their website [18]. In the VisSemantic dataset, the stimuli used in is identical to those reported in [19] with the only difference being that stimuli were written words rather than environmental sounds. Detail of VisSemantic dataset is described as follow.

\section{A. Participants}

Nineteen native right-handed English speakers ( 9 males, 10 females, mean age $=24.4$ years; range $20-37$ years) have participated in this experiment. All had normal or corrected to normal vision and were free of any history of neurological problems. The University of Queensland's Medical Research Ethics Committee approved the study.

\section{B. Stimuli and Experimental Design}

Stimuli were written words depicting 40 concrete nouns; 20 animals and 20 manmade objects. These stimuli were divided into equal groups based on their visual semantic features (animals with or without fur and manmade items predominantly made of metal or not), and further subdivided into animals that did or did not live in Australia and manmade items that were or were not a tool.

There were four conditions in total: visual semantic, encyclopedic, categorical, and a visual perceptual baseline. For each of these 4 conditions there were two question types designed to reflect the properties of each condition. This resulted in a total of eight different trial types.

1) Visual semantic: a) have Fur? b) made of metal?

2) Encyclopedic: a) live in Australia? b) A tool?

3) Categorical: a) living? b) manmade?

4) Visual perceptual baseline: a) fade in? b) fade out?

The eight trial types were blocked, with five trials per block. At the start of each experimental block, participants were presented with one of the 8 questions viewed on a screen at the foot of the scanner bed via a head-coil mounted mirror. The participant was required to make a yes/no button press in response to each presented word to indicate whether it had the attribute related to the question.

Trial duration was $3.5 \mathrm{~s}$ (1.5 $\mathrm{s}$ for each word sequence representation, followed by $2 \mathrm{~s}$ to allow for a response). Word stimuli in conditions $1-3$ were presented in black Arial font on a white background for $1.5 \mathrm{~s}$. The visual perceptual baseline used animated stimuli that faded in or out against the white background of the screen over $1.5 \mathrm{~s}$. Each question was presented for $3 \mathrm{~s}$ at the beginning of each block, giving a total block time of $20.5 \mathrm{~s}$. Blocks were followed by a blank screen, the duration of which randomly alternated between equal multiples of 3, 4, 5, or 6 TR (resolution time, $2.1 \mathrm{~s}$ ). In each of the four scanning sessions, there were two blocks of trials for each question type (i.e., the same question was presented twice in each session), for each of the four conditions. This gave a total of 80 trials ( 16 blocks) per session and 320 trials (64 blocks) over the four sessions. For conditions (1) and (2), blocks comprised either animals (have fur? live in Australia?) or manmade objects (made of metal? a tool?). For conditions (3) and (4), animals and manmade objects were intermixed in each block. The order of the conditions (eight question types) was counterbalanced within and across participants.

\section{Image Acquisition}

Participants were scanned on a Bruker Medspec 4T system equipped with a transverse electromagnetic head coil [20]. Functional images using T2*-weighted EPI sequence for blood oxygen level dependent contrast were acquired $(\mathrm{TE}=$ $30 \mathrm{~ms}, \mathrm{TR}=2,100 \mathrm{~ms}$, and $\mathrm{FOV}=230 \times 230$ ). In a single acquisition, 36 slices were acquired, each $3-\mathrm{mm}$ thick with a 0.6-mm gap between the slices. The first five volumes were discarded to allow for T1 equilibration effects. Between the four sessions, a high-resolution 3D T1-weighted high-resolution image was acquired using an MP-RAGE sequence $(\mathrm{TI}=900 \mathrm{~ms}, \mathrm{TR}=2,300 \mathrm{~ms}, \mathrm{TE}=2.94 \mathrm{~ms}$, $256 \times 256 \times 176$ matrix, and $0.9-\mathrm{mm} 3$ isotropic voxels).

\section{Preprocessing}

Rigid-body motion correction was carried out on all EPI series using INRIalign [21] and a mean realigned image was created for each participant. This mean image was then coregistered to the corresponding structural (T1) image, using SPM5 (Wellcome Trust Centre for Neuroimaging, London). Each individual's T1 image was then normalized to the SPM5 MNI T1 template using the unified segmentation procedure [22]. The resulting spatial normalization parameters were applied to the EPI time series data and resliced to $3 \times 3 \times 3 \mathrm{~mm}$ voxels. Images were then spatially smoothed with a full width half maximum Gaussian kernel of $9 \mathrm{~mm}$

\section{E. Classifiers}

All the data analysis in this study has been carried out using Matlab ${ }^{\circledR}$ (Mathworks, Natick, MA). We used the MVPA toolbox introduced in [23] as the framework to perform brain decoding. Besides of the built-in classifiers of Neural network (NN) and SVM, CRF model reported in [24] was modified to work on the MVPA platform. In this study samples are the voxel BOLD signals and the labels are the mental states: the 4 categories of questions asked.

$\mathrm{NN}$ is a nonlinear directional graphical model consisting of an input layer, one or multiple hidden layers and an output 
layer. A single hidden layer feed-forward neural network (SLFN) was used in this study because an SLFN can form decision boundary with arbitrary shapes when the activation function which defines the transformation of net input is chosen properly for hidden units [25]. Back-propagation training algorithm was used.

An SVM constructs one or multiple hyperplanes in a high or infinite dimensional space, on which samples are mapped as points and discriminated. The task of SVM is to find the separating hyperplane that has largest distance to the nearest sample points. In this paper, a nonlinear SVM with radial basis function kernels as implemented in the LIBSVM toolbox [26]was tested.

CRF is a directional graph model that models probability distribution of the labels globally conditioned on the samples observed [14]. As it directly models the conditional probability, it does not rely on the Markovian assumption and avoids the label bias problem. Instead of the popular Maximum a Posteriori (MAP) algorithm, we employed a marginalization-based parameter learning approach, which was found outperformed likelihood-based algorithms, to train the CRF models and Tree-reweighted Belief Propagation to make the inference [24].

In the VisSemantic study, there was no significant difference in brain activity between answering 'Categorical' question and 'Visual perception baseline' question revealed by SPM analysis. Therefore, three types of CRF models were trained to investigate the predictability based on VisSemantic dataset when different labels were used. CRF-4 models were trained with all four labels were used. CRF-3 models were trained with 'Visual semantic' question and 'Encyclopedic' question were labeled as 1 stimulus. CRF-2 models were designed as binary classifiers to distinguish the mental states of answering the first 3 categories of questions from that of 'visual perceptual baseline' question.

Leave-one-out cross validation (LOOCV) was used to quantify the accuracy achieved by each classifier [27].

\begin{tabular}{cccc}
\multicolumn{5}{c}{ TABLE I: LOOCV RESULT ON HAXBY DATASET } \\
\hline \multicolumn{4}{c}{ Accuracy (\%) } \\
\hline Subject & NN & SVM & CRF \\
\hline 1 & 38 & 60 & 52 \\
\hline 2 & 37 & 57 & 48 \\
\hline 3 & 46 & 64 & 75 \\
\hline 4 & 39 & 68 & $\mathbf{7 9}$ \\
\hline 5 & 41 & $\mathbf{7 3}$ & 76 \\
\hline 6 & 43 & 62 & 70 \\
\hline 7 & $\mathbf{4 7}$ & 64 & 66 \\
\hline 8 & $\mathbf{4 7}$ & 65 & 47 \\
\hline 9 & 37 & 63 & 56 \\
\hline 10 & 34 & 61 & 54 \\
\hline Mean & $\mathbf{4 0}$ & $\mathbf{6 4}$ & $\mathbf{6 2}$ \\
\hline
\end{tabular}

\section{F. Feature Selection}

Feature selection in machine learning refers to extracting predictive information and removing redundant or misleading information from the samples. On the one hand, it enhances the prediction power and increase classification accuracy; on the other, it reduces the dimensionality of the problem and shortens the computational time. In MVPA of fMRI, each voxel is considered as a dimension in the feature space. Irrelevant voxels that contain little information about different conditions only add unrelated noise to the classifier and decrease the performance. Voxels that contain information that is informative, but redundant with respect to other voxels already being considered only add little to accuracy of classification but increase the computational complexity [10].

In this study, feature selection was carried out in the following steps: First, the raw fMRI images were masked to retain the voxels of the brain only; Second, brain regions identified by SPM study to be having significantly different activity for different stimuli were selected; and finally, the statistical map generated by ANOVA was threshold to further reduce the number of voxels used as features [28].

\begin{tabular}{|c|c|c|c|c|c|}
\hline \multirow{2}{*}{ Subject } & \multicolumn{5}{|c|}{ Accuracy (\%) } \\
\hline & $\mathrm{NN}$ & SVM & CRF-4 & CRF-3 & CRF-2 \\
\hline 1 & 25 & 52 & 10 & 34 & 45 \\
\hline 2 & 26 & 55 & 21 & 22 & 52 \\
\hline 3 & 33 & 55 & 27 & 46 & 56 \\
\hline 4 & 20 & 53 & 39 & 43 & 53 \\
\hline 5 & 27 & 53 & 32 & 33 & 45 \\
\hline 6 & 23 & 56 & 22 & 30 & 56 \\
\hline 7 & 31 & 56 & 27 & 40 & 49 \\
\hline 8 & 34 & 53 & 38 & 52 & 66 \\
\hline 9 & 26 & 56 & 30 & 52 & 59 \\
\hline 10 & 25 & 53 & 24 & 38 & 40 \\
\hline 11 & 21 & 53 & 25 & 47 & 42 \\
\hline 12 & 23 & 53 & 15 & 31 & 43 \\
\hline 13 & 24 & 54 & 21 & 38 & 46 \\
\hline 14 & 37 & 56 & 18 & 32 & 52 \\
\hline 15 & 34 & 55 & 43 & 59 & 64 \\
\hline 16 & 23 & 53 & 18 & 32 & 52 \\
\hline 17 & 23 & 54 & 34 & 40 & 49 \\
\hline 18 & 32 & 55 & 29 & 38 & 55 \\
\hline 19 & 17 & 54 & 21 & 37 & 60 \\
\hline Mean & 26 & 54 & 26 & 40 & 52 \\
\hline
\end{tabular}

\section{RESUlTS AND DISCUSSION}

The cross-validation results obtained from three classification methods on the Haxby dataset are tabulated in Table 1 and the boxplot of the results is shown in Fig. 1(a). It can be seen that NN is not comparable to other two methods. SVM has the best overall performance and smallest variance indicating good inter-subject generalization. CRF's overall performance is slightly poorer than SVM but achieved higher accuracy than the highest one of SVM in 3 out of 10 runs. The comparison suggested that CRF has the potential to outperform SVM but proper training samples are required to achieve high inter-subject generality.

For the VisSemantic dataset, the brain regions used in the second step of feature selection include middle frontal gyrus, superior medial frontal, precentral gyrus, posterior middle temporal gyrus, insula, and superior occipital on the right hemiphere, and middle temporal gyrus, inferior frontal gyrus, middle occipital, and inferior occipital on the left hemisphere. The LOOCV results on this dataset are shown in Table 2 and the boxplot is illustrated in Fig. 1(b). Again, NN produced much poor results than SVM. SVM had the best overall performance with 54\% accuracy and very good inter-subject 
robustness. CRF-4 only had comparable accuracy as $\mathrm{NN}$, which was close to 'guessing by chance'. CRF-3 yielded better classification accuracy with $40 \%$ on average than CRF-4. CRF-2 achieved a comparable mean accuracy to SVM but with larger variance. However, CRF-2 generated the highest accuracy $66 \%$ and it had accuracy higher than or equal to the best achieved by SVM (56\%) in 6 out of 19 cross-validation runs. This again demonstrated the potential of CRF and further investigation on feature selection and model training for CRF is of great interest.

The increasing accuracy of CRF models with the decreasing of label space dimension is consistent to the findings of SPM study, in which significant differences in the brain activity patterns were found only between the 'high-selective' questions ('Visual semantic' and 'Encyclopedic') and the 'low-selective' questions ('Categorical' and 'Visual perceptual baseline').

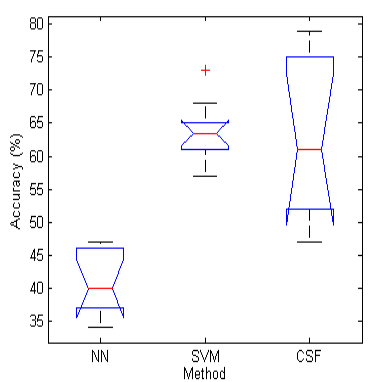

(a) Haxby

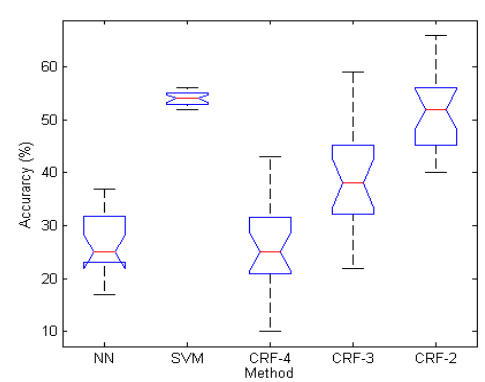

(b) VisSemantic
Fig. 1. Boxplot of the results.

\section{CONCLUSION}

Two well-established pattern classification methods NN and SVM and an emerging classifier CRF were exploited to decode brain mental states from task related functional MRI data in two experimental settings. NN was found inferior to the other two methods. SVM had the best overall performance and best inter-subject generality. When label space was properly defined, CRF yielded comparable results to SVM. The potential of CRF to achieve higher accuracy than SVM demonstrated on both of the datasets is worthy of further investigation.

\section{REFERENCES}

[1] E. P. Duff et al., "Task-driven ICA feature generation for accurate and interpretable prediction using fMRI," Neuroimage, vol. 60, no.1, pp. 189-203, 2012

[2] K. A. Norman, S. M. Polyn, G. J. Detre, and J. V. Haxby, "Beyond mind-reading: multi-voxel pattern analysis of fMRI data," Trends in Cognitive Sciences, vol.10, no.9, pp. 424-430, 2006.

[3] M. Mur, P. A. Bandettini, and N. Kriegeskorte, "Revealing representational content with pattern-information fMRI--an introductory guide," Social Cognitive and Affective Neuroscience, vol. 4, no.1, pp. 101-109, 2009.

[4] J. D. Haynes and G. Rees, "Predicting the orientation of invisible stimuli from activity in human primary visual cortex," Nat Neurosci, vol. 8, no.5, pp. 686-691, 2005.

[5] J. D. Haynes, and G. Rees: "Predicting the stream of consciousness from activity in human visual cortex," Current Biology, 15, (14), pp. 1301-1307, 2005.

[6] M. V. Peelen, A. J. Wiggett, and P. E. Downing, "Patterns of fMRI activity dissociate overlapping functional brain areas that respond to biological motion," Neuron, vol.49, no.6, pp. 815-822, 2006.

[7] M. M. Monti et al., "Willful modulation of brain activity in disorders of consciousness," New England Journal of Medicine, vol. 362, no.7, pp. $579-589,2010$
[8] J. Mourao-Miranda, A. L. Bokde, C. Born, H. Hampel, and M. Stetter, "Classifying brain states and determining the discriminating activation patterns: support vector machine on functional MRI data," Neuroimage, vol. 28, no.4, pp. 980-995, 2005.

[9] M. Misaki, Y. Kim, P.A. Bandettini, and N. Kriegeskorte, "Comparison of multivariate classifiers and response normalizations for pattern-information fMRI," Neuroimage, vol. 53, no.1, pp. 103-118, 2010.

[10] D. D. Cox and R. L. Savoy, "Functional magnetic resonance imaging (fMRI) "brain reading": detecting and classifying distributed patterns of fMRI activity in human visual cortex, "Neuroimage, vol. 19, no. 2. Pt 1, pp. 261-270, 2003.

[11] S. LaConte, S. Strother, V. Cherkassky, J. Anderson, and X. $\mathrm{Hu}$,"Support vector machines for temporal classification of block design fMRI data," Neuroimage, vol. 26, no.2, pp. 317-329, 2005.

[12] T. M. Mitchell et al., "Learning to Decode Cognitive States from Brain Images," Machine Learning, vol. 57, no.1-2, pp. 145-175, 2004.

[13] O. Yamashita, M. A. Sato, T. Yoshioka, F. Tong, and Y. Kamitani, "Sparse estimation automatically selects voxels relevant for the decoding of fMRI activity patterns," Neuroimage, vol.42, no.4, pp. 1414-1429, 2008.

[14] J. D. Lafferty, A. McCallum, and F. C.N. Pereira, "Conditional random fields: probabilistic models for segmenting and labeling sequence data," in Proc. of the 18th Int.Conf. on Machine Learning, 2001.

[15] D. T. Jun Li, "A Probabilistic Model for Discovering High Level Brain Activities from fMRI," Neural Information Processing Lecture Notes in Computer Science, vol. 7062, pp 329-336, 2011.

[16] Y. Wang and J. C. Rajapakse, "Contextual modeling of functional MR images with conditional random fields," IEEE Trans. on Medical Imaging, vol. 25, no.6, pp. 804-812, 2006.

[17] J. Li and D. Tao, A Probabilistic Model for Discovering High Level Brain Activities from fMRI Neural Information Processing; Berlin, Springer-Heidelberg, 2011, pp. 329-336.

[18] G. J. Detre, S. M. Polyn, C. D. Moore, V. S. Natu, B. D. Singer, J. D. Cohen, J. V. Haxby, and K. A. Norman, "The Multi-Voxel pattern analysis (MVPA) toolbox, " Hum Brain Mapp, 2006.

[19] J. Hocking, K. L. McMahon, and G. I. de Zubicaray, "Cortical organization of environmental sounds by attribute, "Hum Brain Mapp, vol. 32, no.5, pp. 688-698, 2011.

[20] J. T. Vaughan et al., "Detunable transverse electromagnetic (TEM) volume coil for high-field NMR," Magnetic Resonance in Medicine MAGN RESON MED, vol. 47, no. 5, pp. 990-1000, 2002.

[21] L. Freire, A. Roche, and J. F. Mangin, "What is the best similarity measure for motion correction in fMRI time series?, "IEEE Trans. on medical imaging, vol. 21, no.5, pp. 470-484, 2002.

[22] J. Ashburner, and K. J. Friston: "Unified segmentation," Neuroimage, vol. 26, no. 3, pp. 839-851, 2005.

[23] J. V. Haxby, M. I. Gobbini, M. L. Furey, A. Ishai, J. L. Schouten, and P. Pietrini, "Distributed and overlapping representations of faces and objects in ventral temporal cortex, "Science, vol.293, no. 5539, pp. 2425-2430, 2001.

[24] J. Domke, "Beating the likelihood:marginalization-based parameter learning in graphical models," in Editor: "Book Beating the likelihood:marginalization-based parameter learning in graphical models."

[25] G. B. Huang, "Learning capability and storage capacity of two-hidden-layer feedforward networks, "IEEE Trans. on Neural Networks, vol. 14, no.2, pp. 274-281, 2003.

[26] C. Chung and C. J. Lin, “ $\{$ LIBSVM $\}$ : A library for support vector machines," ACM Transactions on Intelligent Systems and Technology, vol. 2 , no. 3 , pp. $2: 27: 1-27: 27,2011$.

[27] A. J. O'Toole, F. Jiang, H. Abdi, N. Penard, J. P. Dunlop, and M. A. Parent, "Theoretical, statistical, and practical perspectives on pattern-based classification approaches to the analysis of functional neuroimaging data, " Journal of cognitive neuroscience, vol. 19, no.11, pp. 1735-1752, 2007.

[28] F. Pereira, T. Mitchell, and M. Botvinick, "Machine learning classifiers and fMRI: a tutorial overview," Neuroimage, vol. 45, no.1, Supp 1, pp. S199-209, 2009.

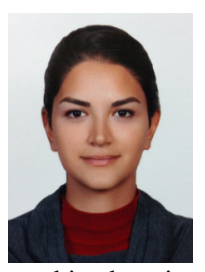

Jeiran Choupan is currently a $\mathrm{PhD}$ candidate with Centre for Advanced Imaging at The University of Queensland, Australia. She did her Bachelor of Science and Engineering (2008) and Master of Science and Engineering in Information Technology at Sharif University of Technology, Iran (2010). Her current research focus is on brain decoding using functional MRI. Her area of expertise is on signal processing and machine learning methods. 


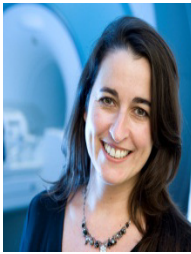

Julia Hocking is a cognitive neuroscientist with a background in psychology (BSc) and cognitive neuropsychology (MSc). She completed her $\mathrm{PhD}$ on object feature integration at the Wellcome Trust Centre for Neuroimaging at University College London, UK. The core theme of her research is on how the brain perceives, organises, recalls and manipulates conceptual knowledge. In 2006, Julia moved to Australia to take up a postdoctoral position at the Centre for Magnetic Resonance, University of Queensland. She currently holds an Australian National Health and Medical Research Council clinical training fellowship to investigate the impact that temporal lobe epilepsy has on memory function, using functional MRI. This work is being carried out at the Centre for Advanced Imaging at the University of Queensland.

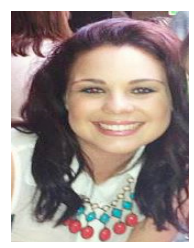

Kori Johnson is a research assistant with a background in psychology (BPsychSc). In 2009 she completed her honours thesis under the supervision of Dr. Julia Hocking at the Centre of Advance Imaging at the University of Queensland. From 2010 to 2012 she worked as a full-time research assistant at the Queensland Institute of Medical Research with an associate research position at the Centre for Advance Imaging, University of Queensland, working on the Twins Neuro-Imaging Genetics Project under the supervision of Dr. Margie Wright and Dr. Katie McMahon. Currently, Kori works as a casual research assistant in the School of Psychology at the University of Queensland under the supervision of Dr.Greig de Zubicaray, working on a number of cognitive fMRI studies, with a particular focus on psycholinguistics.

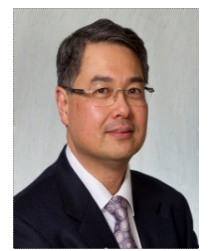

Professor David Reutens was appointed as the inaugural director of the CAI in 2008. Prior to that he was the Professor of Neuroscience at Monash University and Director of Neurology at Southern Health. He is also a clinical neurologist specializing in epilepsy and is a senior staff specialist at the Royal Brisbane and Women's Hospital. He directs the Australian Mouse Brain Mapping Consortium. Professor Reutens' research involves the combination of imaging techniques such as MRI, EEG, transcranial magnetic stimulation and PET to study functions such as memory in the healthy brain, the mechanisms behind diseases such as epilepsy and stroke and how the brain responds to overcome injury. Some of the research studies involve patients with injuries or genetic mutations that affect brain function - these studies are able to provide privileged insights into how the brain works. With his collaborators, he uses imaging to study animal models of disease. Members of his group are working on ways of improving imaging technology such as the development of new diagnostic agents and new mapping methods that may improve the safety of brain surgery.

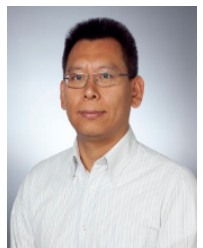

Zhengyi Yang is currently a research fellow working in School of Information Technology and Electrical Engineering at The University of Queensland, Australia. He holds degrees of $\mathrm{PhD}$ from The University of Hong Kong, Master of Science and Bachelor of Engineering from Sichuan University, China. His current research focuses on medical image processing and analysis. 